\title{
Iluminación natural de edificios de oficina
}

Magali Bodart Profesora, Université Catholique de Louvain

Waldo Bustamante Profesor, Pontificia Universidad Católica de Chile

Felipe Encinas Investigador Architecture et Climat, Université Catholique de Louvain
En la búsqueda por controlar la iluminación natural y las inclemencias del clima, se han generado formas de medición basadas en la ubicación geográfica, en los horarios y los estados del tiempo. El uso de métricas dinámicas, que consideren factores en permanente cambio, permite evaluar tipologías representativas de edificios de oficinas construidos en Santiago.

Actualmente, el modelo predominante usado para el análisis de iluminación natural de edificios es el Factor Luz Diurna (FLD), que no considera la orientación ni el clima. A través de él se busca alcanzar valores mínimos recomendados según el destino de uso del recinto. Esto ha conducido al diseño de edificios altamente vidriados, que habitualmente presentan serios problemas de sobrecalentamiento y deslumbramiento en sus espacios interiores. Ambos fenómenos son esencialmente dinámicos y, por tanto, pueden presentar variaciones importantes entre una hora y otra. Los indicadores en que debe basarse el diseño de la iluminación natural en edificios, no solo deben entregar una idea de los valores mínimos, sino que también de los máximos, para dar cuenta de los riesgos de deslumbramiento mencionados. Se trata entonces, de caracterizar la iluminación natural del edificio a través de métricas dinámicas que consideren en el tiempo las variaciones climáticas del lugar. Varios de estos indicadores se han desarrollado en los últimos años. La primera parte de este artículo -escrito por Magali Bodart- presenta estas métricas, su cálculo y representación. A través de un breve ejemplo, se muestra su uso como base para el diseño de edificios de alto estándar energético y medioambiental.

En el segundo segmento, Waldo Bustamante y Felipe Encinas presentan un análisis del comportamiento energético de edificios de oficina en Santiago, sobre el cual se aplican las métricas expuestas anteriormente. Esto, en el contexto de la búsqueda de estrategias de diseño que apunten a resolver problemas de deslumbramiento y de sobrecalentamiento en este tipo de edificios, con el fin de acercarse a estándares de confort con uso eficiente de energía.
In the search for control of natural lighting and weather inclemency, systems of measurement have been generated based on geographical location, times, and weather conditions. The use of dynamic measurements that consider factors in constant change is applied to evaluate representative typologies of office buildings built in Santiago.

\section{Métricas dinámicas}

MÉTRICAS ACTUALES / El Factor Luz Diurna, es un indicador desarrollado hace unos cincuenta años en el Reino Unido (Moon, 1942). Expresa la relación entre la iluminancia horizontal en un punto dentro de un edificio y la iluminancia horizontal exterior simultánea, en un sitio perfectamente claro, para un cielo de diseño $\mathrm{CIE}^{2}$ nublado. Este valor, todavía de uso frecuente -ya sea como un valor objetivo al diseñar un edificio o para evaluar la calidad ambiental de edificios en uso- fue en sus orígenes un valor mínimo a lograr según lo indicado en la ley sobre acceso a la luz (Reinhart, 2006).

El FLD no considera ni el clima en que se ubica el edificio, ni su orientación. La elección de un valor mínimo conduce generalmente a edificios de fachadas altamente vidriadas, especialmente cuando el clima en que se encuentran solo cuenta con algunos días nublados. Además, no ofrece alternativas para una diferenciación respecto de la orientación de la apertura, lo que lleva a fachadas idénticas en todo el edificio y genera contradicción con los principios fundamentales de la arquitectura bioclimática o sostenible.

$\mathrm{Al}$ dejar fuera los aspectos térmicos, el diseño de un edificio basado en la maximización del FLD, ciertamente conducirá a problemas de deslumbramiento y falta de confort lumínico en el edificio. Obligará además al uso extensivo de protecciones solares y, en consecuencia, al uso de iluminación artificial casi permanente, lo que se opone a los objetivos de edificios de alto estándar.

Algunos sistemas de certificación, como LEED ${ }^{3}$ y BREEAM ${ }^{4}$, dan importancia a las posibles vistas hacia el exterior. Si bien es cierto que ello mejora el confort visual de los ocupantes del edificio, este es solo uno de los muchos criterios que lo influencian. Ahora, si los ocupantes tienen una visión hacia el exterior constantemente obstruida por la protección solar, este criterio se vuelve mucho menos importante.

Afortunadamente, algunos arquitectos están conscientes de las limitaciones del Factor Luz Diurna y junto con cumplir con valores mínimos de éste, tratan de evitar la penetración solar directa en ciertas épocas del año (Reinhart, 2006). Este enfoque es, sin duda más interesante que la sola consideración del fLD, pero sigue teniendo la desventaja de no considerar el clima del lugar, aun cuando el acceso al sol se integre al diseño del edificio. Por otra parte, el FLD deja de lado otros aspectos positivos de la penetración de la luz solar en un edificio, como los efectos en la salud y el bienestar de los ocupantes (Hobday, 2006). Este método tampoco considera el tipo de recinto o sus horarios de uso. 
1 Artículo traducido del francés por Waldo Bustamante.

2 Commission Internationale de I'Eclairage. (N. del Ed.)

3 Leadership in Energy and Environmental Design. (N. del Ed.)

4 Building Research Establishment Environmental Assessment Methodology. (N. del Ed.)

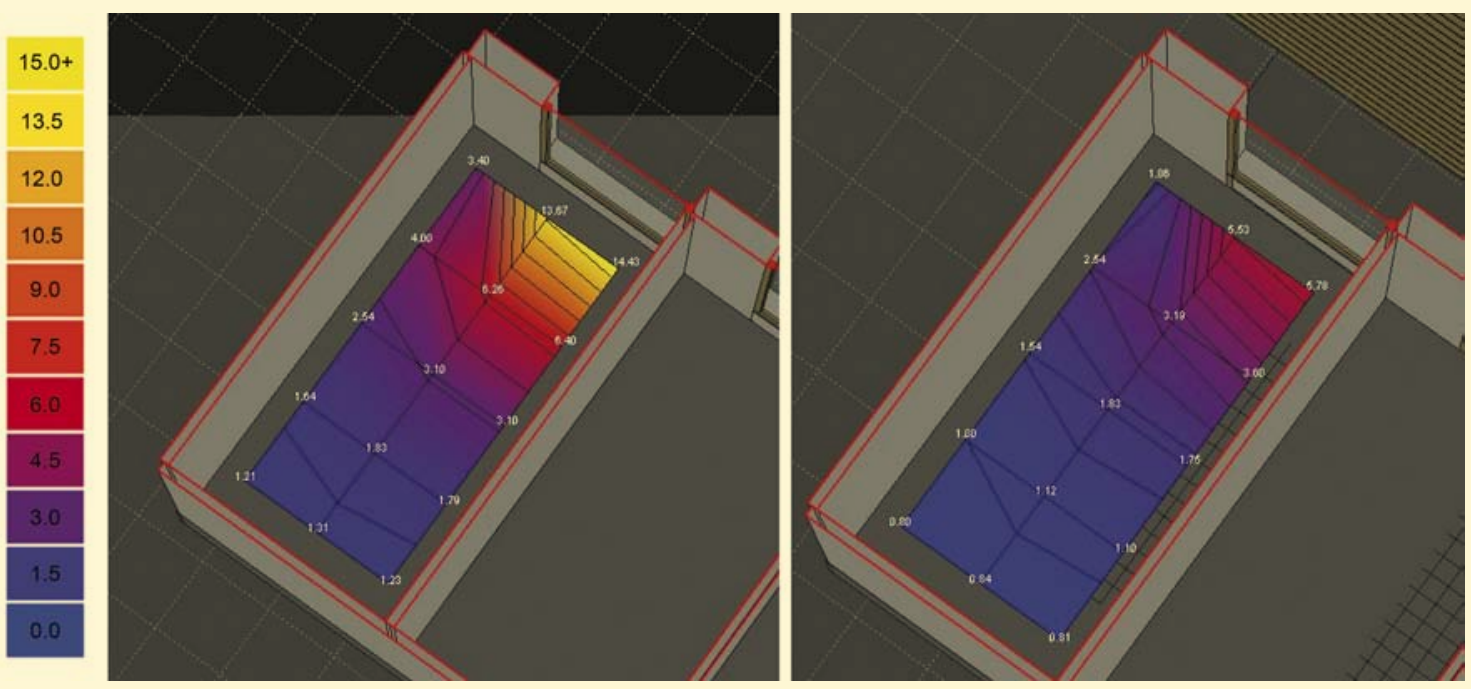

01 FLD de dos recintos orientación sur-poniente en el hemisferio norte, sin y con protección solar. Fuente: elaboración propia

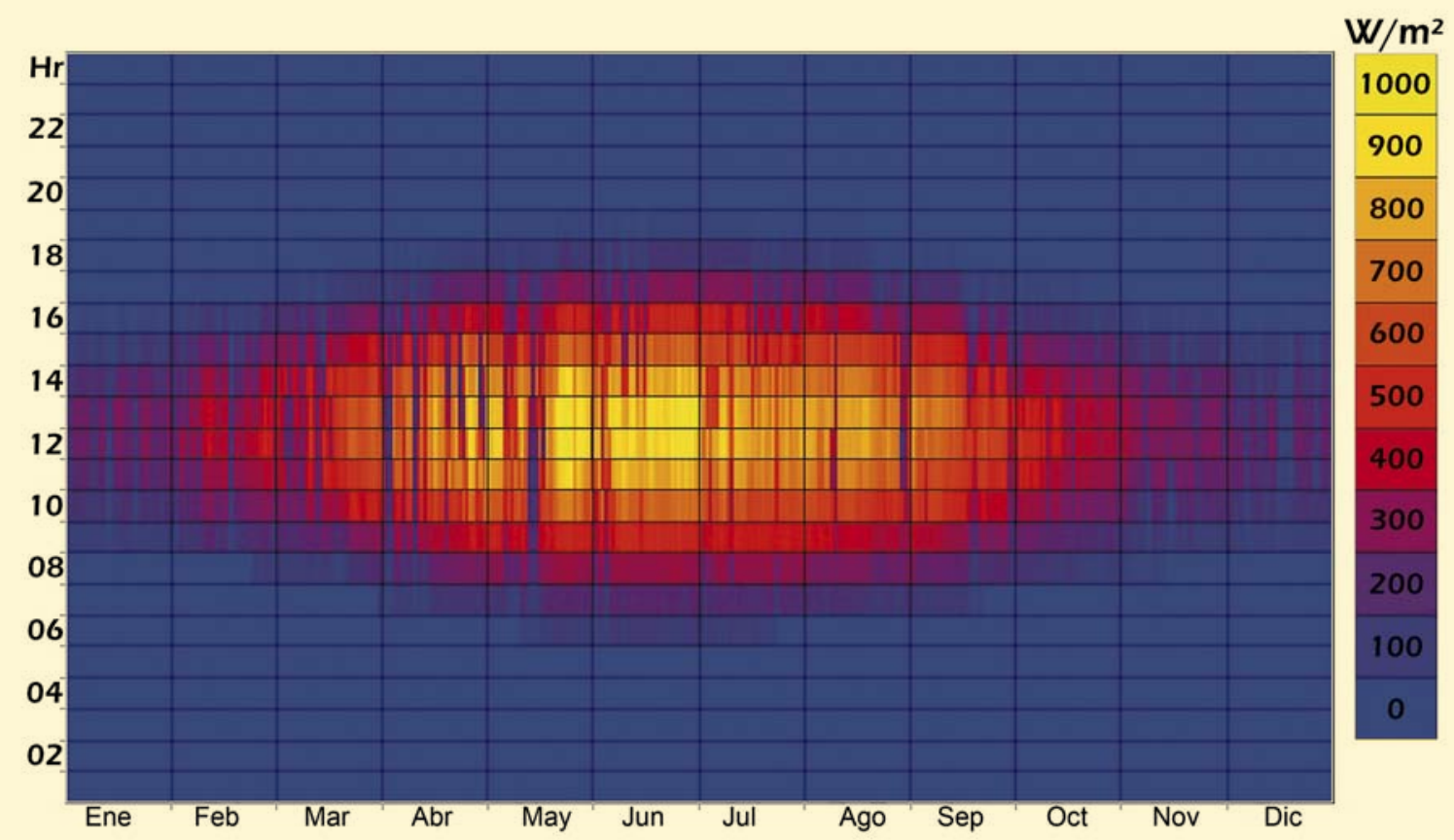

02 Ejemplo de mapa temporal de radiación solar incidente en Roma, Italia. Creado en EcoTecr. Fuente: elaboración propia

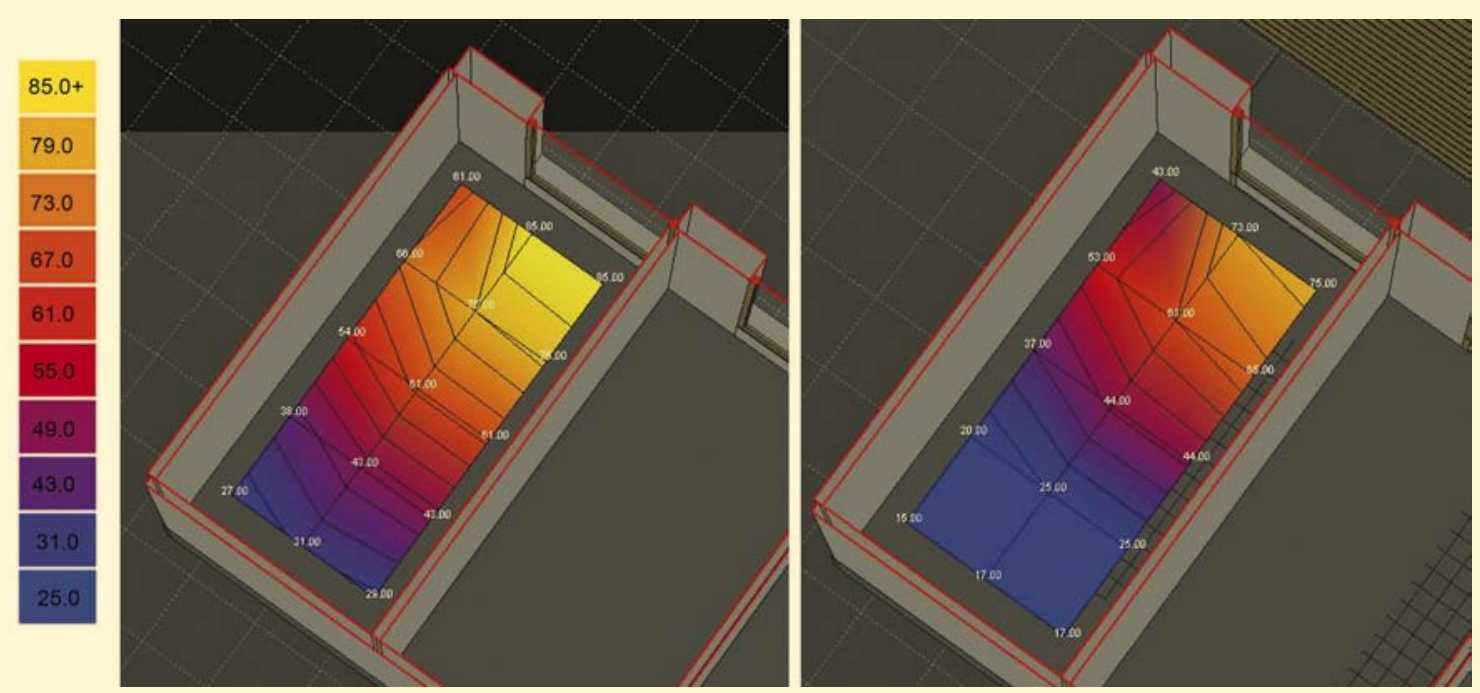

03 DA de dos locales orientación sur-poniente en el hemisferio norte, sin y con protección solar fija. Fuente: elaboración propia 

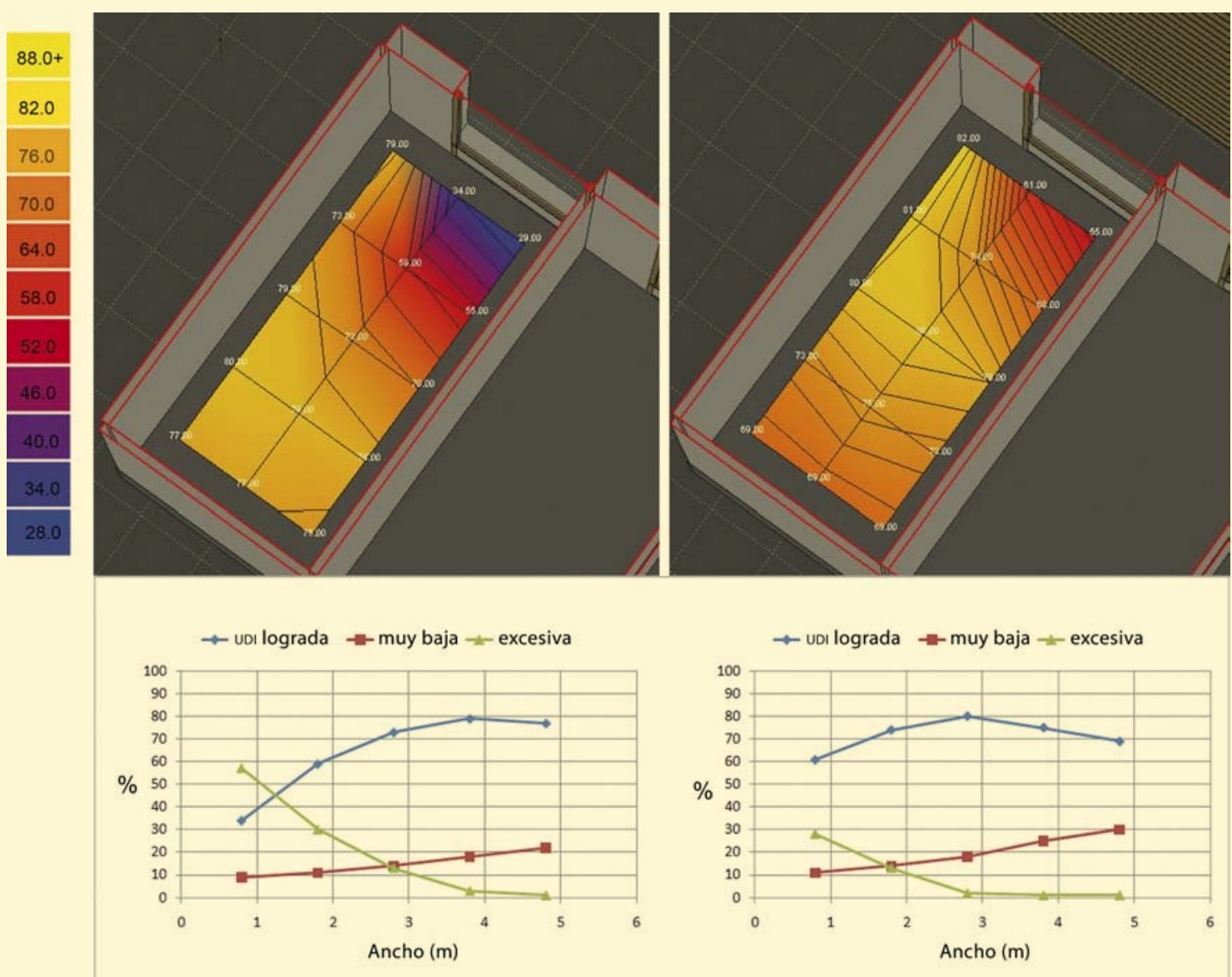

04 uDi para dos locales orientación sur-poniente en el hemisferio norte, sin y con protección solar fija. Fuente: elaboración propia

MÉTRICAS DINÁMICAS / Como alternativa a las métricas utilizadas actualmente, cuyas limitaciones han sido anteriormente expuestas, investigadores del área han desarrollado métricas para el modelamiento dinámico de la luz natural. 5

Esta es una medida calculada a partir de datos climáticos estándares de un lugar. Puede ser, por ejemplo, un valor de iluminancia, de luminancia o de caracterización del deslumbramiento. Estas variables evolucionan durante el día y el año, contrariamente a las métricas estáticas como el FLD.

En iluminación natural, es posible evaluar una métrica dinámica ya sea a través de una simulación o con mediciones en un modelo reducido. El principio es idéntico en ambos casos: a partir de datos meteorológicos, se modela el cielo para cada hora del año y la métrica requerida puede ser entonces calculada o medida en una maqueta (Bodart et al., 2006).

Surge así la pregunta sobre análisis de este valor. De hecho este método da, para cada punto del espacio, su evolución durante el año. Esta evolución es generalmente evaluada en forma horaria, lo que significa obtener 8.760 valores de cada parámetro calculado. Para aportar al diseño y mejorar la iluminación natural del recinto en estudio, la interpretación de estos datos puede ser temporal, espacial o una combinación de ambos.

Si se desea realizar un análisis temporal, es decir, estudiar la evolución de una cierta métrica durante el año, más sencillo es la representación gráfica. Una forma muy visual de hacerlo es la representación en un plano temporal, como lo propone John Mardaljevic (2004).

En un mapa temporal, los días del año están representados en la abscisa x y la hora en la ordenada y. El valor obtenido para la métrica estudiada está dado por su color y leyenda asociada. Para conocer el resultado en cierto momento, simplemente se elije el día en x y la hora en y. Al moverse a través de una paralela a $\mathrm{x}$, se muestra la evolución de la métrica durante el año a cierta hora. Al desplazarse a través de una paralela a y, se observa la evolución de la métrica en cierto día. Un mapa temporal es entonces una forma simplificada de sintetizar un gran número de datos, y representar la evolución de una métrica a través del año.

Una forma de obtener información de un valor adoptado por una métrica en diferentes partes de un edificio es través de un mapa temporal con los puntos estudiados. Si se pretende evaluar solo algunos puntos -lo que es lógico si se quiere alcanzar un análisis espacial preciso-, nos encontraremos con muchos mapas temporales. El análisis de los resultados se vuelve tedioso. Para evitar este problema, se puede proceder de dos maneras. En primer lugar, se puede reducir la evolución del valor de la métrica en un punto de estudio. Esta es la forma utilizada en el cálculo de la Autonomía Dinámica DA (Reinhart, 2006) o de la Iluminancia Natural Útil, UDI (Nabil, 2005). Luego se puede calcular cuántos puntos logran los objetivos fijados, enfoque utilizado por la herramienta Lightsolve (Andersen et al., 2008). 
6 Dynamic Daylight Simulations. http://www.daysim.com. (N. del Ed.)

7 El proyecto Lightsolve busca desarrollar nuevos enfoques en la simulación de la luz día y llenar algunos vacíos críticos de las herramientas disponibles en la actualidad. (N. del Ed.)

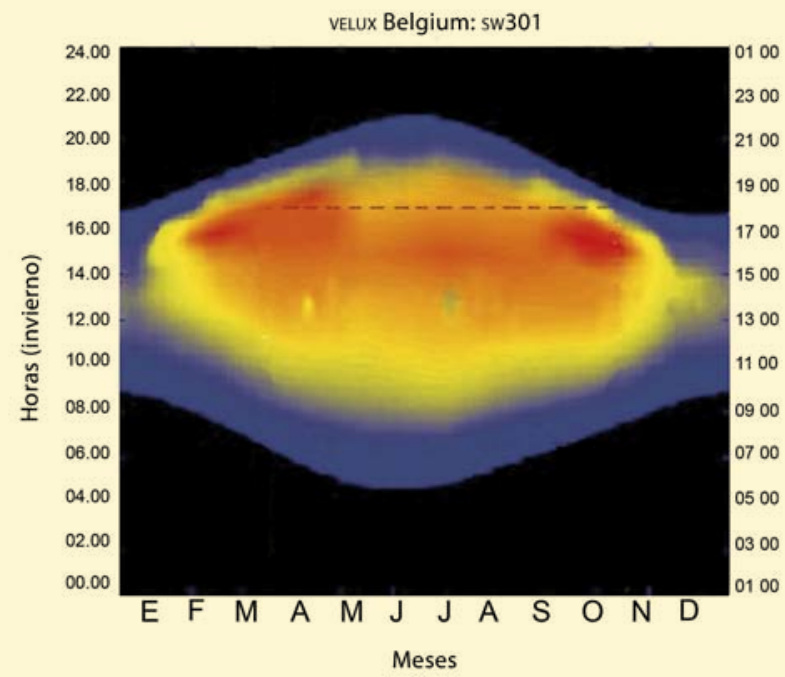

05 Mapa temporal y escala triangular de Lightsolve. Fuente: elaboración propia

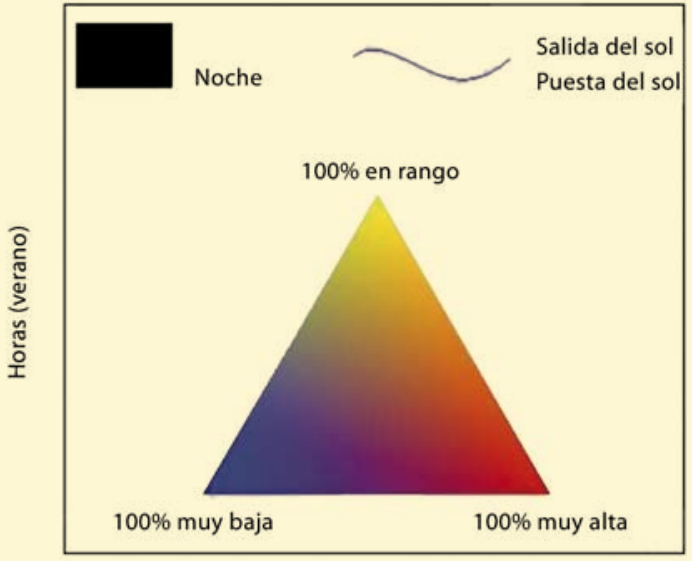

Escala triangular
EjEMPLos / La Autonomía Dinámica de luz diurna es el porcentaje de tiempo durante el cual se mantiene un nivel mínimo de iluminación en el plano de trabajo, solo con luz natural. Para estimar la DA en un punto, se debe calcular la evolución de la iluminación natural a través del año. Una vez conocido este valor, se le compara con un monto de consigna fijado para cada intervalo de tiempo de cálculo -que es en general entre 1 hora y 5 minutos-. Si la iluminación está por encima de la referencia, se puede decir que el local es autónomo en luz natural en el instante considerado. El software DAYsIM ${ }^{6}$ realiza este cálculo.

La autonomía en iluminación natural también puede estimarse a partir de mediciones en maquetas, en combinación con DAYsim (Bodart et al., 2006). Otros dos indicadores derivados de la autonomía dinámica de la iluminación natural han sido propuestos por Zack Rogers (2006). Se trata de la autonomía dinámica continua, que otorga créditos parciales a los valores que están entre 0 lux y el valor de consigna requerido y la autonomía dinámica máxima, que entrega información sobre la proporción de tiempo en que se espera iluminación muy elevada en cierto punto, pudiendo ser fuente de deslumbramiento.

La evaluación a través del indicador UDI -Iluminancia Natural Útil- tiene énfasis en el aspecto espacial. De hecho es una métrica basada en la iluminación en un punto del plano de trabajo. Como su nombre lo indica, su objetivo es establecer si la iluminación en ese plano es útil para el ocupante. El cálculo uDI se basa en los valores umbrales a alcanzar sin sobrepasarlos. Por ejemplo, la iluminación se considera demasiado baja si es inferior a 100 lux, demasiado alta si está sobre 2000 lux y útil si se encuentra entre estos dos valores. El cálculo de uDI dispone de tres métricas, que indican el porcentaje del tiempo durante el cual la luz en el plano de trabajo es demasiado débil, útil y demasiado alta. $\mathrm{Al}$ igual que la $\mathrm{DA}$, la uDI puede ser fácilmente representada en forma espacial. La Iluminancia Natural Útil también se puede calcular utilizando DAYsIM.

La orientación elegida en Lightsolve $e^{7}$ pone acento en lo temporal. Al igual que para el cálculo UDI, se fijan objetivos cuantitativos. Se define un área de interés y se calcula, para cada momento del año, el porcentaje del espacio que cumple con los objetivos. Al representar los resultados en forma de mapa temporal -con una escala triangular- es posible informar al usuario, en un solo gráfico, el porcentaje de puntos demasiado o muy poco iluminados. Luego, en un único gráfico se tiene información espacial -porcentaje del espacio que responde a los objetivos- y temporal -evolución de este valor durante el año-.

CONSIDERACIONES / Para el análisis de iluminación natural en edificios, se debiera abandonar el uso del FLD en beneficio de las métricas dinámicas. Últimamente se han desarrollado diferentes métricas dinámicas, las que se pueden evaluar informáticamente o a partir de mediciones en maquetas. Aunque queda mucho por hacer por parte de los investigadores, tal como establecer estándares para estas métricas, es esencial que los diseñadores las usen a partir del presente, pues si se toma en cuenta la dinámica del clima local, la ocupación del edificio y el uso de protectores solares móviles, la iluminación natural se podrá optimizar en el diseño de un edificio de alto estándar.

\section{Magali Bodart}




\section{Edificios de oficinas en Santiago:}

Tipologías / En el análisis integrado de desempeño técnico y lumínico de edificios de oficinas en Santiago se aplicó un procedimiento estadístico de análisis de clústeres $^{9}$ para seleccionar tipologías de edificios representativos del parque existente en la Región Metropolitana. Se analizaron cincuenta edificios construidos en los últimos cinco años en diversas comunas de la región, obteniendo dos tipologías, una de ellas $-68 \%$ de la muestra- presenta fachada completamente transparente, con doble vidriado de tipo selectivo. El segundo tipo cuenta con fachada combinada de muros de hormigón armado con ventanas de vidriado simple. En ambos se evidencian severos problemas de deslumbramiento y de sobrecalentamiento, aunque el edificio con fachada completamente vidriada es el más crítico al respecto. Este artículo presenta el análisis de un edificio con muro cortina, en que se considera su desempeño térmico e iluminación natural. Asimismo se proponen diversas estrategias de mejoramiento.

COMPORTAMIENTO TÉRMico Y LUMÍNICO / En la búsqueda por resolver problemas de sobrecalentamiento y deslumbramiento, la respuesta arquitectónica que ha entregado el mercado nacional ha estado caracterizada por la inclusión de vidrios de tipo reflectante o selectivo, en desmedro de la protección solar exterior. Estos vidrios -que en comparación con un vidriado simple claro- logran reducir en un sesenta por cientola transmisión total de calor, han sido promovidos desde la perspectiva de la eficiencia energética. Sin embargo, estos sistemas no garantizan por si solos el alcanzar estándares adecuados de confort térmico y lumínico. Deben, por el contrario, estar acompañados de estrategias de enfriamiento pasivo, tales como protección solar y ventilación nocturna combinada con inercia térmica.

Se propone una metodología que integra el análisis de confort térmico y lumínico para el diseño de edificios de oficina, desde una perspectiva dinámica que integra la variabilidad climática y la capacidad de adaptación de las personas a su ambiente inmediato. Por medio de esta, se pretende evaluar el impacto de la incorporación de estrategias de enfriamiento pasivo en el comportamiento térmico y lumínico en los edificios, por medio de tres indicadores:

Grados-hora de sobrecalentamiento (EN15251, 2007) en base al modelo de confort adaptativo basado en estudios de usuarios reales de edificios de oficina. Luminancia $\left(\mathrm{cd} / \mathrm{m}^{2}\right)$ como indicador del riesgo de deslumbramiento y excesivos contrastes en momentos críticos del año. ${ }^{10}$

Autonomía de Luz Diurna DA e Iluminancia Natural Útil UDI. Indicadores de niveles excesivos de iluminación natural, los cuales pueden estar asociados a deslumbramiento y ganancias de calor indeseadas. ${ }^{11}$

COMPARACIÓN / Los edificios con muro cortina cuentan con fachadas completamente vidriadas independiente de su orientación, con doble vidriado de tipo selectivo -de nombre comercial Sunergy Azur Glaverbel-, transmisión lumínica de 0,50 y transmisión solar de 0,41.

En la figura 1 se muestran los resultados relacionados a los problemas de sobrecalentamiento y las demandas de energía para enfriamiento en este edificio. En ella, el centro de cada burbuja representa la medida de sobrecalentamiento en los pisos 5, 10 y 15, expresado en grados-hora -valor promedio para las cuatro oficinas de cada piso-. El área de la burbuja corresponde a la desviación estándar de los valores en estos recintos. De este modo, el mejor caso corresponde a las burbujas ubicadas más abajo en la escala y que son de menor tamaño ${ }^{12}$.

Las simulaciones muestran los problemas de sobrecalentamiento en el edificio original -tal cual ha sido diseñado-, los que decrecen al incorporar protección solar exterior, independiente o en combinación con ventilación nocturna. En este último caso, la demanda de energía de enfriamiento alcanza a aproximadamente $40 \mathrm{kWh} / \mathrm{m}$ - año, lo que está lejos de representar un edificio energéticamente eficiente según estándares internacionales. En el caso de la tipología que representa al segmento de edificios con fachada de hormigón y ventanas de vidriado simple, se alcanza una demanda de enfriamiento aproximada de 12 $\mathrm{kWh} / \mathrm{m}$ - año, que se obtuvo al aplicar protección solar exterior con ventilación nocturna, doble vidriado y aislación térmica exterior de $50 \mathrm{~mm}$ en los muros de hormigón armado.

La figura 2 presenta los resultados de simulaciones de luminancia para el solsticio de verano, a las 12 horas en día despejado. Se observa que la luminancia del muro cortina envolvente del recinto bordea los límites recomendados ${ }^{13}$, mientras que el cielo del recinto presenta luminancia

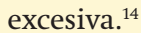

La figura 3 muestra la iluminancia natural útil uDI en el edificio. Se observa riesgo de deslumbramiento hacia la fachada norte y en menor intensidad, hacia el poniente. La presencia de doble vidriado selectivo no necesariamente permite evitar los problemas de confort lumínico.

CONSIDERACIONES / En Santiago, los edificios de oficinas con fachadas completamente vidriadas presentan problemas de confort térmico y lumínico, lo que se expresa en muchas horas de sobrecalentamiento en los recintos y problemas de deslumbramiento en ciertos momentos del año. El uso de vidrio selectivo, aun en doble vidriado hermético, no asegura la solución a estos problemas. El uso del doble vidriado está orientado principalmente a mejorar el comportamiento térmico en el período de calefacción.

De esta manera, un mejor comportamiento térmico y lumínico en un edificio de oficinas en Santiago se logra mediante el manejo adecuado del porcentaje de superficie vidriada, combinado con estrategias de diseño como la protección solar y el uso de ventilación nocturna con inercia térmica, lo que se complementa, a su vez con aislación térmica exterior en muros. ARQ

Waldo Bustamante, Felipe Encinas

\section{Bibliografía}

Andersen, Marilyne; Kleindienst, Sian; Yi, Lu; Lee, Jaime; Cutler, Barbara y Magali Bodart. "An intuitive daylighting performance analysis and optimization approach". Building Research and Information, № 36(6). Routledge, Nueva York, 2008. / Bodart, Magali; Deneyer, Arnaud y V. Gilbert. "Validation of the Belgian single-patch sky and sun simulator”. Building and Environment. № 43. Elsevier V.B., Maryland, 2008. / Bodart, Magali; Deneyer Arnaud; De Herde, André y Peter Wouters. "Design of a new single patch sky and sun simulator". Lighting Research and Technology. Vol. 38. SAGE Publications, Londres, 2006. | EN15251. Parámetros del ambiente a considerar para el diseño y evaluación de la eficiencia energética de edificios incluyendo la calidad del aire interior, condiciones térmicas, iluminación y ruido. Comité Europeo de Normalización CeN, Bruselas, 2007. / IEs. The IESNA Lighting Handbook. Reference \& Application. M.S. Rea Ed., Illuminating Engineering Society of North America, Nueva York, 2000. / Hobday, Richard. The light revolution, health, architecture and the sun. Findhorn Press, Dyke, 2006. / Kittler, Richard; Darula, Stanislav y David MacGowan. The critical luminance causing glare in interiors. Light and Lighting Conference with Special Emphasis on Led's and Solid Stale Lighting. Cie-Hungary, Budapest, 2009. / Mardaljevic, John. "Spatio-temporal dynamics of solar shading for a parametrically defined roof system”. Energy and Buildings, № 36. Elsevier V.B., Maryland, 2004. / Moon, Parry y Domina Spencer. Illumination from a non-uniform sky. Illuminating Engineering Society, Nueva York, 1942. / Nabil, Azza y John Mardaljevic. "Useful Daylight Illuminance: A new paradigm to access daylight in buildings". Lighting Research \& Technology. № 37(1). SAGE Publications, Londres, 2005. / Reinhart, Christoph; Mardaljevic, John y Zack Rogers. "Dynamic daylight performance metrics for sustainable building design." Leukos. Vol.3, $\mathrm{N}^{\circ} 1$. Illuminating Engineering Society of North America, Nueva York, julio de 2006. / Reinhart, Cristophy Oliver Walkenhorst. "Dynamic RADIANCE-based daylight simulations for a full-scale test office with outer venetian blinds". Energy and Buildings, № 33. Elsevier V.B., Maryland, 2001. / Rogers, Zack. Daylighting metric development using daylight autonomy calculations in the sensor placement optimization tool. Architectural Energy Corporation, Boulder, 2006. http://www.archenergy.com/SPOT/download.html. 
8 Estudio desarrollado en el contexto del proyecto FoNDECY 1090602 . a cargo de los investigadores

Waldo Bustamante y Felipe Encinas, Enfriamiento ambiental de edificios de oficina a través de ventilación nocturna, Pontificia Universidad Católica de Chile, 2009.

9 Procedimiento estadístico que parte de un conjunto de datos que contienen información sobre una muestra de entidades e intenta organizarlas en grupos relativa-

mente homogéneos, a los que llama clüsteres. (N. del Ed.)
${ }^{10}$ Estimado con el software Radiance.

" Estimados con los softwares Radiance Y DASIM.

12 Las demandas de enfriamiento y los grados-hora de sobrecalentamiento se han estimado con TAs, software de simulación térmica en régimen dinámico.

13 No mayor a $2.500 \mathrm{~cd} / \mathrm{m}^{2}$ de acuerdo a Kittler et ál, 2009 .

14 No debería exceder las $700 \mathrm{~cd}$ $\mathrm{m}^{2}$ de acuerdo a IES, 2000.

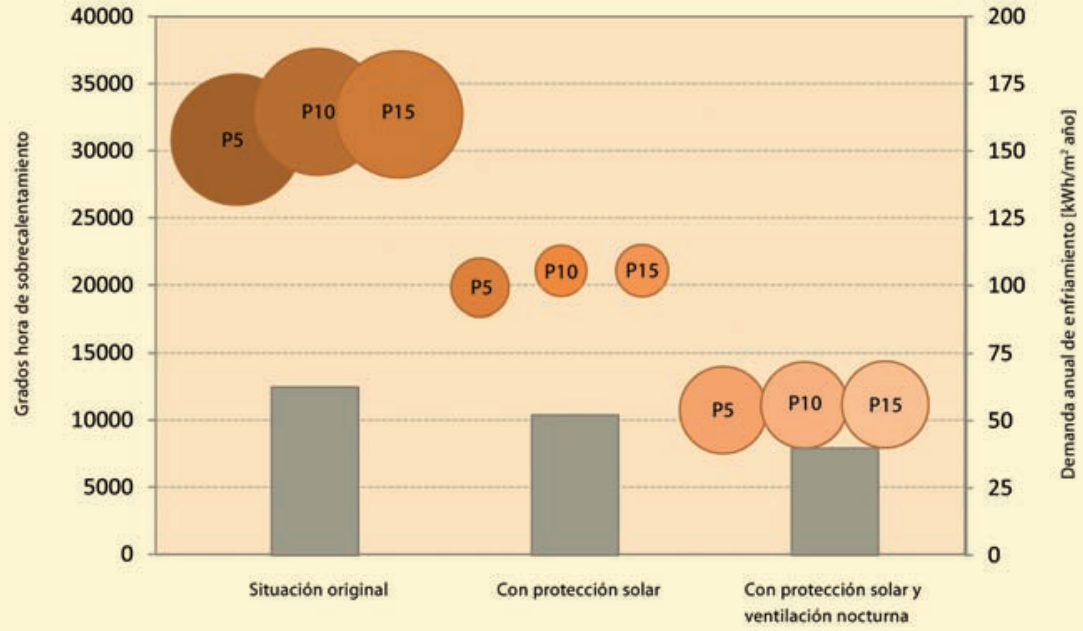

01 Simulación de grados-hora de sobrecalentamiento en base al modelo de confort adaptativo y demanda anual de enfriamiento; para los pisos 5, 10 y 15. Fuente: elaboración propia

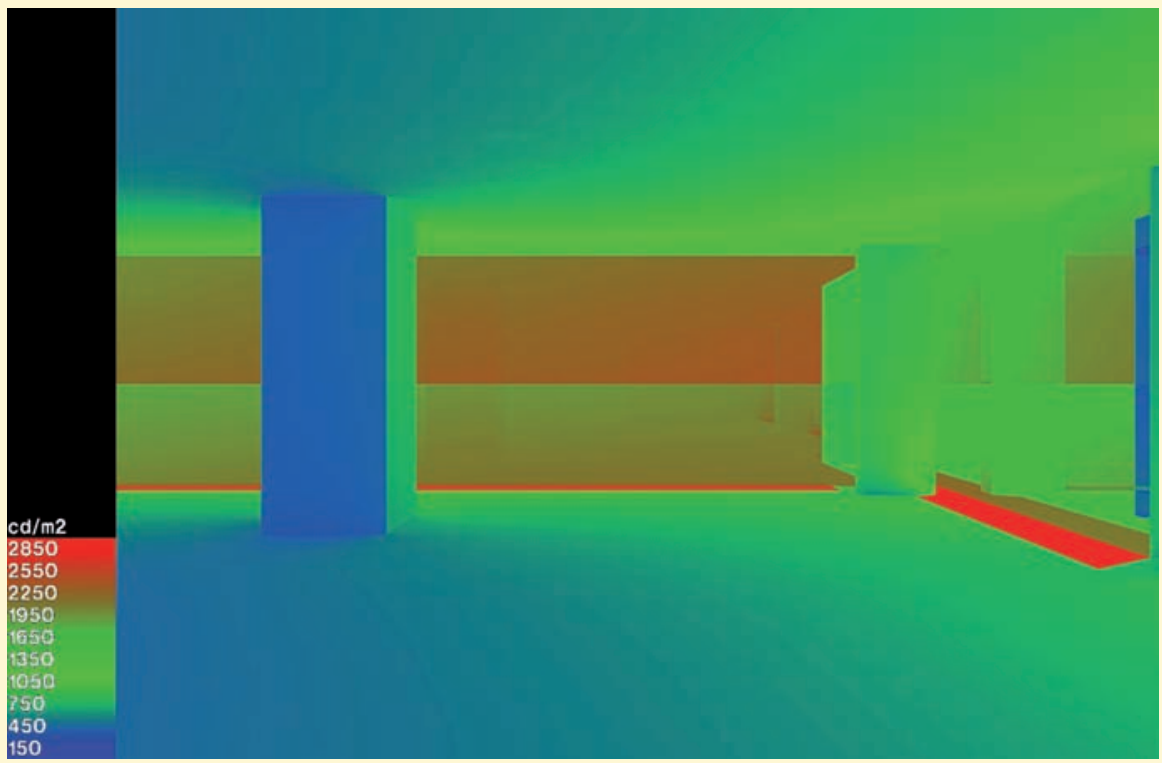

02 Resultados de análisis de luminancia [cd/m²], 12 horas; 21 de diciembre, cielo despejado. Visualización con colores falsos. Fuente: elaboración propia

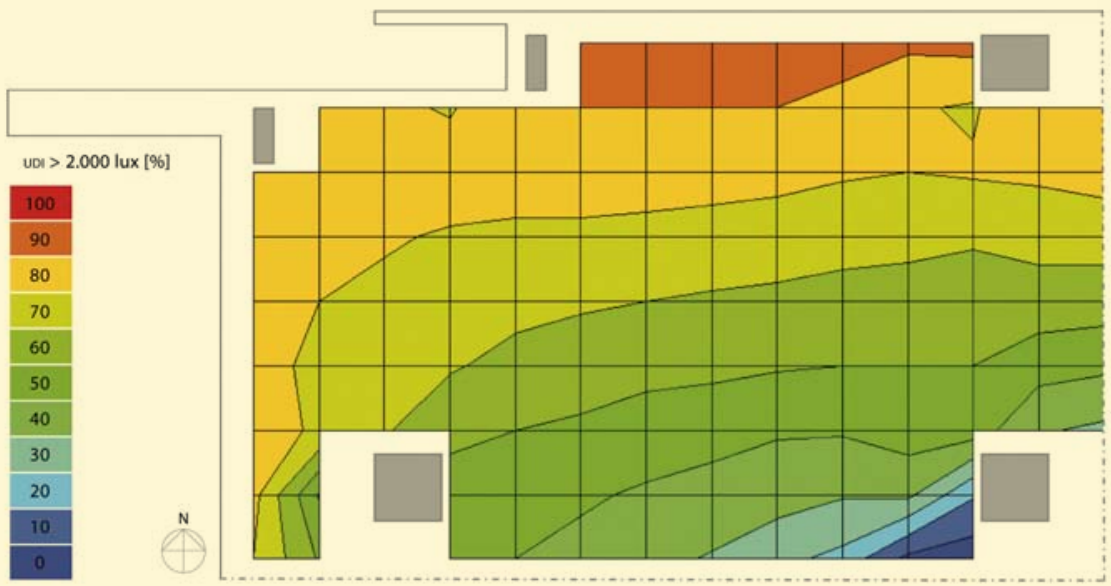

03 uDı para el rango mayor a 2.000 lux. Líneas de contorno de uDi > 2.000 lux casa 10\%. Fuente: elaboración propia 